\title{
Cardiac Dysfunction in Adult Patients with Traumatic Brain Injury: A Prospective Cohort Study
}

\author{
Chakradhar Venkata, MD and Jan Kasal, MD, FCCM
}

Background: There are limited data regarding the development of myocardial dysfunction after a traumatic brain injury (TBI). We investigated incidence, risk factors, and prognostic importance of cardiac dysfunction in adult patients admitted to the intensive care unit (ICU) after a moderate to severe TBI.

Methods: Prospective observational study of consecutive patients admitted to neuro-trauma ICU with moderate to severe TBI from August 2014 to June 2015.

Results: A total of 46 patients were included. Patients' mean $( \pm \mathrm{SD})$ age was $44.7( \pm 20.7)$ years and mean Glasgow Coma Scale value was $5.6( \pm 3)$. Motor vehicle accident was the most common mechanism of TBI, with subdural and subarachnoid hemorrhages as the most common pathologies. Cardiac dysfunction developed in 6 of 46 (13\%) patients. Patients with cardiac dysfunction had higher prevalence of diabetes mellitus $(50 \%$ vs. $10 \%, P=0.03)$ and higher proportion of electrocardiogram abnormalities $(83 \%$ vs. $27 \%, P=$ 0.02 ) compared to the patients without cardiac dysfunction. Mean Glasgow Coma Scale scores were not significantly different between patients who developed cardiac dysfunction from those who did not (5.5 vs. $5.6, P=0.95)$. Requirement for vasopressor support $(33.3 \%$ vs. $40 \%, P=1.0)$ and median ventilator days $(5.2$ vs. $4.7, P=0.9)$ were similar between patients with and without cardiac dysfunction. There were no significant differences in hospital lengths of stay ( 12.3 vs. I 3.8 days, $P=0.34$ ) and hospital mortality $(33 \%$ vs. $17.5 \%, P=$ 0.58 ) between the two groups.

Conclusions: Cardiac dysfunction occurs in patients after moderate to severe TBI, with mild to moderate reduction in left ventricular ejection fraction. Patients who developed cardiac dysfunction after TBI had a higher prevalence of diabetes mellitus and higher proportion of abnormalities in electrocardiograms. Development of cardiac dysfunction was not associated with adverse clinical outcomes.

Keywords: Cardiac dysfunction; Traumatic brain injury

$\mathrm{C}$ ardiac complications after acute neurological injury are common and are well studied in patients with subarachnoid hemorrhage and stroke. ${ }^{1,2}$ However, there are limited data about myocardial dysfunction in patients with traumatic brain injury (TBI). A retrospective study $^{3}$ found that cardiac dysfunction occurred in $22 \%$ of patients (31 out of 139 patients) after an isolated TBI. They also found that the abnormal echocardiogram was independently associated with increased in-hospital mortality. However, it is plausible that the occurrence of cardiac dysfunction could have been overestimated due to their study design. A subsequent prospective study from the same

Corresponding Author: Chakradhar Venkata, MD, 62S S New Ballas Rd, Suite 7020, St. Louis, MO 63I4I USA, Tel: (3I4) 25I-6486, Fax: (3I4) 25I- 4I55, Email: chakradhar.venkata@gmail.com investigators found that $22 \%$ (7 out of 32) of patients developed left ventricular systolic dysfunction after a moderate to severe TBI. ${ }^{4}$ These findings contradict results from two recent studies that did not find left ventricular systolic dysfunction in a cohort of patients with moderate to severe TBI. ${ }^{5,6}$

Due to the paucity of data about the incidence of cardiac dysfunction after a TBI, we decided to conduct a prospective cohort study evaluating the occurrence of cardiac dysfunction after a moderate to severe TBI. We hypothesized that cardiac dysfunction occurs after a TBI and is associated with the 


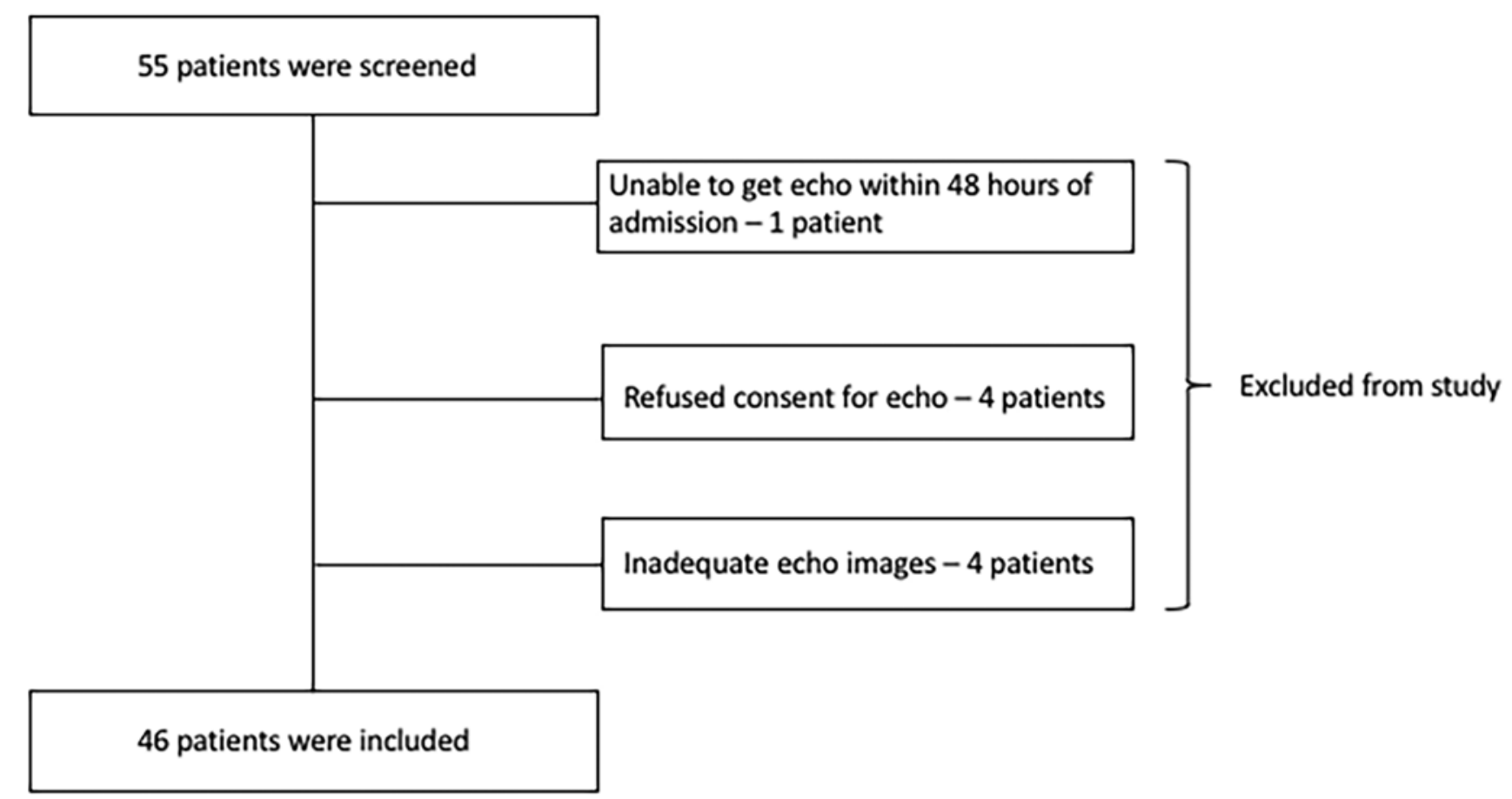

Figure 1. Patient enrollment flow diagram.

severity of brain injury. We further sought to evaluate risk factors for the development of cardiac dysfunction and its impact on clinical outcomes.

\section{Methods}

This prospective observational study was conducted at Mercy Hospital in Saint Louis, Missouri. Mercy Hospital is an 859bed hospital and a level I trauma center for Saint Louis County, with more than 300 head-trauma admissions per year. The study was approved by the institutional review board of the Mercy Hospital, Saint Louis, which provided a waiver of informed consent due to the observational nature of the study. Although a waiver of informed consent was awarded, all patients or their next of kin were asked for permission before performing the echocardiography examination. Patients or their next of kin were informed about the study purpose and were offered an option to decline the echocardiography examination. All adult patients admitted to the 16-bed neurotrauma intensive care unit (ICU), between August 2014 and June 2015 were screened daily for the admission diagnosis of TBI. This ICU operates as a closed ICU model, staffed by neuro-intensivists with critical care fellows and residents. Patients were treated in accordance with Brain Trauma Foundation guidelines. The study investigators neither requested any tests nor interfered in the management of these patients. We excluded patients who declined the echocardiography examination. Other exclusion criteria were history of congestive heart failure, history of cardiomyopathy, and inability to obtain adequate quality echocardiography images from at least three imaging planes.

TBI was defined as an alteration in brain function or other evidence of brain pathology caused by an external force. ${ }^{7}$ The severity of TBI was classified based on the Glasgow Coma Scale (GCS) value. TBI with a GCS of $\geq 13$ was considered mild, 9-12 moderate, and $\leq 8$ was considered severe. ${ }^{8}$ Cardiac dysfunction was defined as the presence of either left ventricular systolic dysfunction (left ventricular ejection fraction $<55 \%$ ) or regional wall motional abnormalities diagnosed by echocardiography.

The following data were abstracted from the patient's electronic medical records: age, ethnicity, sex, medical comorbidities, post-resuscitation admission Glasgow coma scale, head findings on computed tomography (CT), electrocardiogram (ECG) and comprehensive echocardiogram findings (when available), use and duration of mechanical ventilation, incidence of acute kidney injury according to acute kidney injury network criteria, ${ }^{9}$ vasopressor duration, ICU and hospital lengths of stay, and functional outcome at the time of discharge from the hospital. The Acute Physiology, Age and Chronic Health Evaluation (APACHE) III score values and functional status at hospital discharge were abstracted from the APACHE outcomes database of our institution. ${ }^{10}$ Head abbreviated injury scale (HAIS) and injury severity score (ISS) values were abstracted from the institutional trauma database. The worst cardiovascular and respiratory Sequential Organ Failure Assessment (SOFA) ${ }^{11}$ scores were calculated for days 1,3 , and 5 of a patient's ICU stay. In the calculation of the SOFA score, the worst values for each parameter in the 24-hour period were used. Cardiac enzymes (troponin T, CK, CK-MB) and pro-BNP laboratory values were collected when available.

\section{Echocardiography Details}

Three senior critical care fellows acquired goal-oriented 
critical care echocardiography examinations under the supervision of the study investigator (CV) who is a National Board of Echocardiography Testamur. All three fellows had expertise in image acquisition and underwent formal training in critical care ultrasonography during the fellowship, which included one month of structured echocardiography rotation and a 12-hour symposium of critical care ultrasonography. All patients had echocardiograms performed within 48 hours of admission to the ICU. A standardized protocol was used for the image acquisition and collection of data points for the cardiac ultrasound exam (Supplemental material: S1 Echo Protocol). CX50 portable echocardiography machine (Phillips, Netherlands) was used for performing the echocardiography examinations. The images were stored digitally in cine loop format. All echocardiogram examinations were reviewed offline by the senior investigator (JK), who is a National Board of Echocardiography Testamur with more than 10 years of clinical expertise and scholarly contributions in the field of critical care echocardiography. All echocardiography examinations were analyzed blindly to the clinical data. Left ventricular ejection fraction (LVEF) was measured using Simpson's method of discs when possible. ${ }^{12}$ In patients where the endocardial border was not clearly visualized on either apical four- or apical two-chamber views to accurately use the Simpson's method, the LVEF was assessed qualitatively by visual estimation. Right ventricle (RV) size was assessed according to standard recommendations. ${ }^{12} \mathrm{RV}$ size was evaluated for mild, moderate, or severe dilation based on $\mathrm{RV} / \mathrm{LV}$ area ratio (normal $<0.6$, mild 0.6-0.9, moderate 1.0, severe $>1.0$ ). RV systolic function and LV diastolic function were not measured. Some of the patients enrolled in the study had comprehensive echocardiograms ordered by treating physicians due to clinical indication, and those data were used when available. These echocardiograms were acquired by certified sonographers and interpreted by certified cardiologists who were unaware of the patients' enrollment into the study.

\section{Statistical Analysis:}

Data were summarized as mean (standard deviation) (SD), median (interquartile range) (IQR), or percentages. We used unpaired student's t-test to compare continuous variables with normal distribution and Wilcoxon test for skewed distribution. For comparison of categorical variables, we used chi-square test if the number of elements in each cell was 5 or higher and Fisher's exact test otherwise. Comparison was made between patients with and without cardiac dysfunction with respect to clinical outcomes. We used multiple logistic regression analysis to determine independent risk factors associated with the development of cardiac dysfunction and their relative impact. Univariate analysis was done to identify the candidate variables for multiple logistic regression analysis. Covariates with a low $P$ value $(\leq 0.1)$, or ones that were thought to have strong biologic associations, were entered into the model. A $P$ value of $<0.05$ was considered statistically significant. The JMP statistical software (version 13.0, SAS Institute Inc., Cary, NC, USA) was used for all data analyses.

\section{Results}

Between August 2014 and June 2015, there were 55 patients screened for enrollment into the study, and of these, 9 patients were excluded due to various reasons (Figure 1). A total of 46 patients were included in the study, of whom $36(78 \%)$ were men. The patients' mean $( \pm \mathrm{SD})$ age was $44.7( \pm 20.7)$ years, and a majority of them were Caucasians (42 out of 46, $91.3 \%)$. Patients' mean $( \pm \mathrm{SD})$ post-resuscitation admission Glasgow Coma Scale value was $5.6( \pm 3)$. The mechanism of TBI was mostly due to motor vehicle accident and fall. Results showed $59 \%$ of the patients (27 out of 46 ) had isolated TBI, and the rest had various associated orthopedic injuries and intraabdominal injuries, with the mean body Abbreviated Injury Scale score of 3.2 (0.4). In patients with associated body trauma, the most common orthopedic injuries were rib fractures; none had spinal cord injury. A majority of patients had severe TBI (37 out of 46 patients, $80.4 \%$ ), and traumatic subdural and subarachnoid hemorrhages were the most common intracranial pathologies noted on CT scan. Of the study population, one-fourth (12 out of $46,26 \%$ ) underwent decompressive craniectomy (Table 1). Electrocardiograms were done in 35 patients on the day of admission, and 13 patients $(37 \%)$ had abnormal findings. A majority of these findings were nonspecific ST-T wave changes (8 patients). These include T-wave flattening, ST repolarization changes, and minimal ST segment depressions. Other abnormal ECG findings included right bundle branch block (3 patients), left ventricular hypertrophy (1 patient), and prolonged QT interval (1 patient). Cardiac biomarkers were not routinely checked, and the median troponin $\mathrm{T}$ value was $0.01 \mathrm{ng} / \mathrm{mL}$ in 24 of 46 patients (reference range $<0.04$ $\mathrm{ng} / \mathrm{mL}$ ). A total of six patients had elevated troponins; two patients had mild elevation (troponin $\mathrm{T}<0.1$ and $>0.04 \mathrm{ng} /$ $\mathrm{mL}$ ); and the other four had troponin values $>0.1 \mathrm{ng} / \mathrm{mL}$. Table 1 lists details of pre-existing medical comorbidities, use of beta-blocker, and afterload reduction agents prior to the trauma admission.

Echocardiograms were performed within the first 48 hours of admission to the ICU, with a mean (SD) time to echocardiogram after ICU admission of 20.3 (10) hours. Most were goaloriented echocardiograms performed by critical care physicians (31 out of 46, 67\%). LVEF was estimated via Simpson's method of discs in $32(69.6 \%)$ patients, and qualitatively via visual estimation in $14(30.4 \%)$ patients. Six out of 46 patients had developed cardiac dysfunction (13\%), of whom four had mildly decreased LVEF, and two patients had moderately decreased LVEF. Patients with depressed LV systolic function had a global decrease in LVEF, and none had regional wall motion abnormalities. Five patients had right ventricular dilation, and three patients had mild pericardial effusion. Table 2 summarizes the echocardiography related data.

We did not find any association between the severity of TBI and the development of cardiac dysfunction. Mean GCS scores were not significantly different between patients who 
Table 1. Demographics and clinical characteristics of 46 patients admitted to ICU with moderate to severe traumatic brain injury.

\begin{tabular}{|c|c|}
\hline Variable & Value \\
\hline Age (years), mean (SD) & $44.7(20.7)$ \\
\hline Male sex, $\mathrm{n}(\%)$ & $36(78)$ \\
\hline Caucasian, n (\%) & $42(91.3)$ \\
\hline GCS, mean (SD) & $5.6(3)$ \\
\hline APACHE III score, mean (SD) & $54.6(26.8)$ \\
\hline Hypertension, n (\%) & $11(24)$ \\
\hline Diabetes mellitus, n (\%) & $7(15.2)$ \\
\hline Coronary artery disease, n (\%) & $5(10.8)$ \\
\hline Beta-blocker use prior to admission, $\mathrm{n}(\%)$ & $7(15.2)$ \\
\hline Afterload reduction agent use prior to admission, $\mathrm{n}(\%)$ & $6(13)$ \\
\hline Isolated TBI, n (\%) & $27(58.7)$ \\
\hline \multicolumn{2}{|l|}{ TBI severity, $\mathrm{n}(\%)$} \\
\hline Moderate & $9(19.6)$ \\
\hline Severe & $37(80.4)$ \\
\hline \multicolumn{2}{|l|}{ Type of TBI } \\
\hline Subdural hemorrhage, $\mathrm{n}(\%)$ & $11(24)$ \\
\hline Subarachnoid hemorrhage, $\mathrm{n}(\%)$ & $11(24)$ \\
\hline Contusion, n (\%) & $8(17.4)$ \\
\hline Parenchymal hemorrhage, $\mathrm{n}(\%)$ & $4(8.6)$ \\
\hline Other*, n (\%) & $12(26)$ \\
\hline \multicolumn{2}{|l|}{ Mechanism of TBI } \\
\hline Motor vehicle accident, $\mathrm{n}(\%)$ & $28(60.9)$ \\
\hline Fall, n (\%) & $14(30.4)$ \\
\hline Fire arm injury, $\mathrm{n}(\%)$ & $3(6.5)$ \\
\hline Assault, n (\%) & $1(2.2)$ \\
\hline Intracranial pressure monitoring, n (\%) & $10(21.8)$ \\
\hline Craniectomy, n (\%) & $12(26)$ \\
\hline Injury severity score, mean (SD) & $19.1(9.8)$ \\
\hline Head Abbreviated Injury Scale, mean (SD) & $3.3(1.6)$ \\
\hline \multicolumn{2}{|c|}{$\begin{array}{l}\text { *Other injuries include: diffuse axonal injury, epidural hemorrhage, intraventricular } \\
\text { hemorrhage, concussion, skull fractures and extensive pneumocephaly. }\end{array}$} \\
\hline \multicolumn{2}{|c|}{$\begin{array}{l}\text { Abbreviations: ICU, Intensive care unit; SD, Standard deviation; GCS, Glasgow coma scale; } \\
\text { APACHE, Acute Physiology, Age, and Chronic Health Evaluation; TBI, Traumatic brain injury. }\end{array}$} \\
\hline
\end{tabular}

developed cardiac dysfunction and those who did not (5.5 vs. 5.6, $P=0.95)$. The HAIS and ISS were also not different between the two groups (Table 3). Although mean APACHE III scores were higher for patients who developed cardiac dysfunction compared to patients without cardiac dysfunction, it did not reach statistical significance (68.7 vs. $52.4, P=$ 0.08). Patients with cardiac dysfunction had a higher prevalence of diabetes mellitus compared to those without cardiac dysfunction ( $50 \%$ vs. $10 \%, P=0.03)$. Patients with cardiac dysfunction also had higher proportion of ST-T wave abnormalities on ECG ( $83 \%$ vs $27 \%, P=.02)$. Among the six patients who developed cardiac dysfunction, three patients had non-specific ST-T wave changes, one patient had prolonged QT interval (QT - $424 \mathrm{msec}$, QTc - $464 \mathrm{msec}$, heart rate $83 / \mathrm{min}$ ), one had left ventricular hypertrophy related changes, and one patient had normal ECG. Cardiac biomarkers were not frequently checked, hence, we could not estimate the difference between the two groups in univariate analysis. Cardiovascular and respiratory SOFA scores (on ICU days 1, 3, and 5) were similar between patients who developed cardiac dysfunction and those who did not. Presence of cardiac dysfunction was not associated with any adverse clinical outcomes (Table 3). In the multivariate logistic regression model, the presence of diabetes mellitus remained a risk factor of development of cardiac dysfunction after adjusting for APACHE III score (Odds ratio 7.28, 95\% 
confidence interval $1.02-55.5 ; P=0.04)$. Due to the limited number of outcome events (i.e., development of cardiac dysfunction) in the study population, we could not adjust for other variables including age and GCS in the multivariate analysis.

\section{Discussion}

In our prospective study of 46 patients with moderate to severe TBI, six patients (13\%) had developed cardiac dysfunction, four patients had mildly reduced LVEF, and two patients had a moderate reduction in LVEF. The severity of TBI as assessed by GCS score was not associated with the development of cardiac dysfunction. Diabetes mellitus was associated with the development of cardiac dysfunction, and patients with cardiac dysfunction had a higher proportion of ECG abnormalities. Development of cardiac dysfunction was not associated with any adverse clinical outcomes.

The existing data about cardiac dysfunction after a TBI is limited to very few studies, with divergent results about the incidence and its effect on clinical outcomes. In a retrospective study of 139 patients with isolated TBI, in patients who had an echocardiogram performed within 2 weeks of their hospital course, 31 patients $(22 \%)$ were found to have evidence of cardiac dysfunction, as defined by depressed LV systolic function or presence of regional wall motional abnormalities. ${ }^{3}$ They also found that an abnormal echocardiogram was independently associated with increased in-hospital mortality. Echocardiography was not a standard part of the management in their study, and echocardiograms were requested at the discretion of the attending intensivist. As the authors pointed out in their discussion, intensivists would have ordered echocardiograms for a specific reason; hence, their study population could have had a high preclinical probability for cardiovascular dysfunction. In a small prospective study of 49 patients with moderate to severe TBI, none had developed depressed LVEF, and four patients (8\%) had regional wall motional abnormalities with preserved LVEF. $^{6}$ Although up to one-third of patients in this study had elevated troponin, and about half of the patients had ECG

Table 2. Echocardiographic data of 46 patients admitted to ICU with traumatic brain injury.

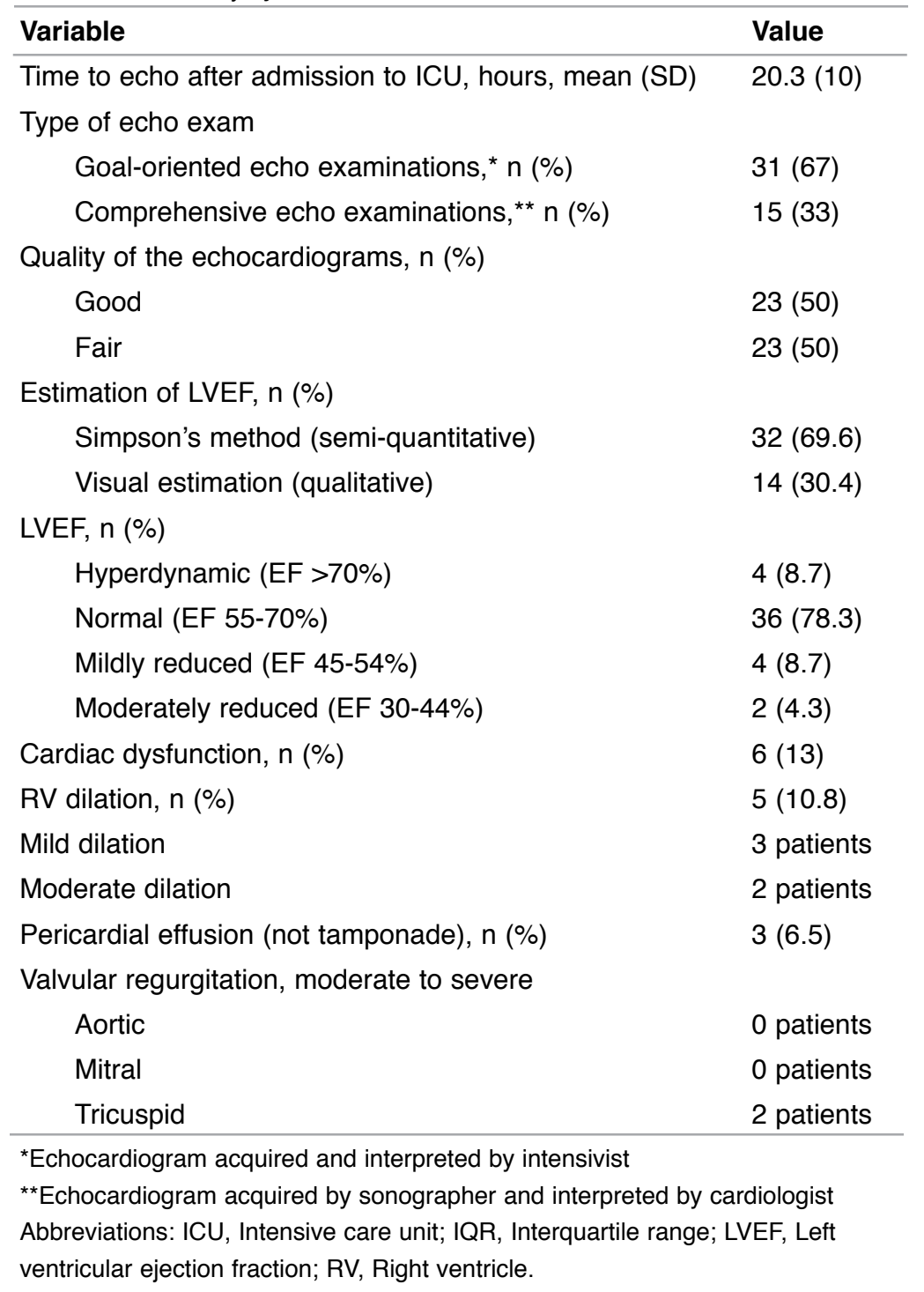


Table 3. Comparison of demographic and clinical outcome data for patients with and without cardiac dysfunction.

\begin{tabular}{|c|c|c|c|}
\hline Variable & $\begin{array}{l}\text { Patients with cardiac } \\
\text { dysfunction }(\mathrm{N}=6)\end{array}$ & $\begin{array}{l}\text { Patients without } \\
\text { cardiac dysfunction } \\
(\mathrm{N}=40)\end{array}$ & $P$ value \\
\hline Age, mean (SD) & $51.8(23.3)$ & $43.7(20.4)$ & 0.37 \\
\hline APACHE III score, median (SD) & $68.7(19.7)$ & $52.4(27.3)$ & 0.08 \\
\hline GCS, mean (SD) & $5.5(3)$ & $5.6(3.1)$ & 0.95 \\
\hline ISS, mean (SD) & $16.6(6)$ & $19.4(10.2)$ & 0.54 \\
\hline Head AIS, mean (SD) & $3.2(1.1)$ & $3.3(1.6)$ & 0.94 \\
\hline Coronary artery disease, n (\%) & $2(33.3)$ & $3(7.5)$ & 0.12 \\
\hline Diabetes mellitus, n (\%) & $3(50)$ & $4(10)$ & $0.03^{*}$ \\
\hline Systemic hypertension, n (\%) & $1(16.7)$ & $10(25)$ & 1.0 \\
\hline Use of beta-blocker or afterload reducing agent, $n(\%)$ & $2(33.3)$ & $8(20)$ & 0.59 \\
\hline EKG changes, $n(\%)$ & $5(83.3)$ & $8(27.6)(\mathrm{N}=29)$ & $0.02^{*}$ \\
\hline Peak troponin $\mathrm{T}, \mathrm{ng} / \mathrm{mL}$, median (IQR) & $0.01-0.03$ & $0.01-0.09(n=18)$ & 0.52 \\
\hline Day 1 cardiovascular SOFA score, median (IQR) & $0.5(0-1.5)$ & $1(0.25-3)$ & 0.2 \\
\hline Day 3 cardiovascular SOFA score, median (IQR) & $1(1-4)$ & $1(1-3)(n=37)$ & 0.46 \\
\hline Day 5 cardiovascular SOFA score, median (IQR) $(\mathrm{n}=36)$ & $1(0-1)(n=5)$ & $1(0-1)(n=31)$ & 0.62 \\
\hline Day 1 respiratory SOFA score, median (IQR) & $2(0.75-4)$ & $2(0-3)$ & 0.62 \\
\hline Day 3 respiratory SOFA score, median (IQR) $(n=23)$ & $2(2-2)(n=2)$ & $1(1-3)(n=21)$ & 0.61 \\
\hline Day 5 respiratory SOFA score, median (IQR) $(n=17)$ & $2(2-2)(n=3)$ & $3(1.75-3)(n=14)$ & 0.25 \\
\hline Ventilator days, median (IQR) & $5.2(2.4-8.8)$ & $4.7(1.6-10.6)$ & 0.9 \\
\hline Development of acute kidney injury, n (\%) & $1(16.7 \%)$ & $10(25 \%)$ & 1.0 \\
\hline Vasoactive medication use, $\mathrm{n}(\%)$ & $2(33.3)$ & $16(40)$ & 1.0 \\
\hline Vasopressor duration, hours, median (IQR) $(n=18)$ & $29.5(24-35)(n=2)$ & $40.5(15-78.7)(n=16)$ & 0.62 \\
\hline ICU length of stay, days, median (IQR) & $6.9(4.6-11.3)$ & $7.5(2.3-14.5)$ & 0.8 \\
\hline Hospital length of stay, days, median (IQR) & $12.3(6.4-14.6)$ & $13.8(8.1-19.9)$ & 0.34 \\
\hline ICU mortality, n (\%) & $2(33)$ & $6(15)$ & 0.27 \\
\hline Hospital mortality, n (\%) & $2(33)$ & $7(17.5)$ & 0.58 \\
\hline
\end{tabular}

*Significant $P$ values

"Laboratory reference ranges for troponin T values - normal: $<0.04 \mathrm{ng} / \mathrm{mL}$, mild elevation: $0.04-0.1 \mathrm{ng} / \mathrm{mL}$, moderate/severe elevation:

$>0.1 \mathrm{ng} / \mathrm{mL}$.

Abbreviations: SD, Standard deviation; APACHE, Acute Physiology, Age, and Chronic Health Evaluation; GCS, Glasgow Coma Scale; ISS, Injury Severity Score; AIS, Abbreviated Injury Scale; SOFA, Sequential Organ Failure Assessment; IQR, Interquartile range; ICU, Intensive care unit.

abnormalities, none had developed depressed LVEF. Study authors postulated that the younger age of the patients in their study could have protected against significant myocardial injury. Another case-control study of 20 patients with severe TBI also did not find depressed LVEF after a TBI. ${ }^{5}$ In a recent prospective study of 32 patients with moderate to severe TBI, the study investigators performed echocardiograms within a day of TBI and followed up with repeat echocardiogram twice after the initial study, over the course of 7-9 days. ${ }^{4}$ Seven out of $32(22 \%)$ patients in that study with moderate to severe TBI developed LV systolic dysfunction, and the LV systolic function recovered within one week. In our prospective study of 46 patients with moderate to severe TBI, we found a lower incidence $(13 \%)$ of patients who developed LV systolic dysfunction. We did not follow these patients longitudinally to confirm recovery of myocardial function. Further studies including a more significant number of patients involving multiple institutions are needed to estimate the incidence of cardiac dysfunction after a TBI.

Incidence of cardiac dysfunction after TBI varied significantly among existing studies, ranging from $0 \%$ to $50 \% 0^{3-6,13,14}$ This discrepancy could be attributed partly to differences in the study design, sample size, heterogeneity in the definition of cardiac dysfunction and differences in the methods of estimating left ventricular systolic function. Two studies from single institution reported that $22 \%$ of patients with TBI had evidence of cardiac dysfunction, despite significant differences in the study design, age of the patient population, severity of TBI and echocardiographic methods for estimating LV 
systolic function (Simpson's method versus fractional shortening). ${ }^{3,4}$ Another study from Egypt reported the presence of abnormal echocardiogram in $42 \%$ of patients with severe $\mathrm{TBI} ;{ }^{13}$ however, the LVEF was assessed by linear methods including fractional shortening and Teichholz method. Fractional shortening is a linear method of estimating LV systolic function and is prone to errors in the presence of segmental wall motion abnormalities or if the measurements are not taken perpendicular to the LV cavity. ${ }^{12}$ Teichholz method is also a linear method of estimating LVEF, which assumes excessive geometrical simplification of LV shape, and it also has additional limitations similar to that of fractional shortening; hence it is not a recommended method to measure LVEF. ${ }^{15}$ Stress cardiomyopathy (Takotsubo pattern) is common after neurological injury ${ }^{16,17}$ in which wall motion abnormalities involving left ventricular basal segments could lead to erroneous estimation of LV systolic function by the linear methods. We assessed LVEF by Simpson's method of discs, or via visual estimation (eyeball method) when Simpson's method could not be used due to poor endocardial border visualization. Qualitative (visual) assessment of LV systolic function is the most commonly used method, and its assessments are consistent with quantitative echocardiographic assessment and nuclear scanning studies when performed by experienced physicians. ${ }^{18,19}$ Simpson's method of discs uses volumetric measurements of LV cavity in two views and is the suggested method for estimating LVEF as recommended by the American Society of Echocardiography. ${ }^{12}$ Future studies addressing myocardial dysfunction in TBI patients should use standardized methods to quantify LVEF.

Systemic complications after neurological injury were thought to be associated with the severity of neurological injury. ${ }^{20}$ In a prospective study of 32 patients with moderate to severe TBI, lower GCS score at admission was one of the risk factors for development for systolic dysfunction. ${ }^{4}$ However, we did not find any association between GCS and the development of cardiac dysfunction. Similar to our findings, in the study by Prathep et al., ${ }^{3}$ distribution of abnormal echocardiograms by GCS severity was equal among all the GCS categories: $24.4 \%$ in severe TBI, $20 \%$ in moderate TBI, $19.6 \%$ in mild TBI. In contrast, HAIS of 4-6 was found to be associated with abnormal echocardiogram in their analysis. Mean HAIS in our study population was 3.3, and we did not find significant differences in HAIS values between patients who developed cardiac dysfunction and those who did not develop cardiac dysfunction. It is possible that GCS may not be a good predictor for the development of cardiac dysfunction in TBI patients due to its imperfect correlation with the actual extent of TBI. ${ }^{21}$ Younger age is reported to be a risk factor for the development of systolic dysfunction after moderate to severe TBI, possibly due to increased myocardial catecholamine responsiveness in young people. ${ }^{4}$ Our study population is composed of older patients with a mean age of 44 years, and we did not find a significant age difference between patients who developed cardiac dysfunction and those that did not develop cardiac dysfunction. Presence of diabetes mellitus was found to be a risk factor for the development of cardiac dysfunction in our study. This is an interesting finding and requires further exploration in future studies.

Cardiac dysfunction after TBI was reported to be a poor prognostic factor. ${ }^{3,13}$ We measured various clinical outcomes including the worst cardiovascular and respiratory SOFA scores, need for vasopressor use, requirement for mechanical ventilation, ventilator days, ICU and hospital lengths of stay, and mortality. We did not find any association between the development of cardiac dysfunction and adverse clinical outcomes. Our results contrast with those of Prathep et al., ${ }^{3}$ who found that the presence of an abnormal echocardiogram is associated with increased hospital mortality. This finding could be due to their study design open to selection bias, as the echocardiograms were ordered at the discretion of attending physicians, up to 2 weeks after the injury. It is plausible that attending physicians requested echocardiograms when there was a clinical indication (e.g., shock); hence, these patients may have had higher mortality due to the underlying condition. Hasanin et al. ${ }^{13}$ reported that a neurogenic cardiac injury score (comprising of troponin elevation, abnormal echocardiogram and systemic hypotension) greater than one was associated with increased mortality in severe TBI patients. This score needs further validation before considering a prognostic marker after TBI. In our study, patients who developed myocardial dysfunction had only mild to moderate reduction in LVEF, and none had severe depression in LV systolic function. This finding, along with the small sample size, may explain the lack of association between the presence of myocardial dysfunction and adverse clinical outcomes.

Our study has several limitations. Due to the observational nature of the study, we could not control for all confounding factors that could have been present in the critically ill patients at different times. We have obtained echocardiograms within first 48 hours of ICU admission but could have missed detection of cardiac dysfunction that might have developed later. We chose this period as the existing literature suggests myocardial dysfunction develops early in the course after a neurological injury. ${ }^{5,22}$ We used two different methods to estimate LVEF in our study: Simpson's biplane method (quantitative) and visual estimation method (qualitative), which could have led to misclassification of the outcome. However, all goal-oriented echocardiograms were interpreted blindly to the clinical data by a single expert physician, which minimized the possibility of inter-observer variability. We also included data from comprehensive echocardiograms when available, and these echocardiograms were acquired by certified sonographers and reviewed by board-certified cardiologists. We only focused on LV systolic function and did not measure LV diastolic function. Because of the small sample size, our analyses were not adequately powered to determine statistically significant differences between the groups. We excluded nine patients from the study for various reasons. Although unlikely, this group potentially could have differed from the larger cohort and influenced overall results. 
Table S1. Echo protocol

\section{A. Views}

1. Parasternal long axis - high depth

2. Parasternal long axis - medium depth

Identify structures

Assess LV size and function, RV size and function, pericardium

Screen for AR and MR (with color doppler)

3. Parasternal short axis

Aortic valve level.

Identification of structures

Screening for TR and AR (color)

Mitral valve level

Papillary muscle level

LV size and function

RV size and function

Pericardial space

4. Apical

Apical 4 chamber - high depth

Screen for presence of pericardial /pleural fluid

Apical 4 chamber - medium depth

Identify structures

LV size and function, RV size and function, pericardium

$\mathrm{RV}$ : LV size ratio estimation

Screen for TR and MR (color Doppler)

5. Subcostal 4 chamber

Identify structures

LV size and function, RV size and function, pericardium

$\mathrm{RV}: \mathrm{LV}$ size ratio

Screen for TR and MR (color)

6. Subcostal inferior vena cava

Assess IVC diameter and document respiratory variation (2D, M-mode)

\section{B. Data points}

- LV systolic function (ejection fraction) estimation: visual (in 5\% increments) and semi-quantitative (Simpson's method).

- LV systolic function categories: hyperdynamic $>70$, normal 55-70, mildly reduced $45-54$, moderately reduced $30-44$, severely reduced $<30$

- Regional wall motion abnormalities

- RV function (normal, reduced)

- RV size (normal, dilated - mild/moderate/severe)

- Record valvular regurgitation (moderate, severe) - Aortic, Mitral, Pulmonary, Tricuspid valves

- Pericardial effusion (none, small, moderate, large)

- Tamponade (yes/no)

\section{Quality of the study}

Good - All images obtained are of diagnostic value.

Fair - Most views were obtained, and most of them are diagnostic

Suboptimal - One or few views were obtained that are diagnostic

Poor - None or few views that were obtained are diagnostic

LV, Left ventricle; RV, Right ventricle; AR, Aortic regurgitation; MR, mitral regurgitation; TR, Tricuspid regurgitation; IVC, Inferior vena cava 


\section{Conclusions}

In this prospective study of adult patients admitted to ICU after a moderate to severe TBI, cardiac dysfunction occurred in $13 \%$ of patients, and its development was not associated with the GCS score. Patients with cardiac dysfunction had mild to moderate reduction in LVEF. Patients who developed cardiac dysfunction had a higher prevalence of diabetes mellitus and a higher proportion of ECG abnormalities. Development of cardiac dysfunction was not associated with adverse clinical outcomes.

\section{References}

1. Dimant J, Grob D. Electrocardiographic changes and myocardial damage in patients with acute cerebrovascular accidents. Stroke. 1977;8(4):448-455.

2. Mayer SA, Lin J, Homma S, et al. Myocardial injury and left ventricular performance after subarachnoid hemorrhage. Stroke. 1999;30(4):780-786.

3. Prathep S, Sharma D, Hallman M, et al. Preliminary report on cardiac dysfunction after isolated traumatic brain injury. Crit Care Med. 2014;42(1):142-147.

4. Krishnamoorthy V, Rowhani-Rahbar A, Gibbons EF, et al. Early Systolic Dysfunction Following Traumatic Brain Injury: A Cohort Study. Crit Care Med. 2017;45(6):1028-1036.

5. Cuisinier A, Maufrais C, Payen JF, Nottin S, Walther G, Bouzat P. Myocardial function at the early phase of traumatic brain injury: a prospective controlled study. Scand J Trauma Resusc Emerg Med. 2016;24(1):129.

6. Serri K, El Rayes M, Giraldeau G, Williamson D, Bernard F. Traumatic brain injury is not associated with significant myocardial dysfunction: an observational pilot study. Scand J Trauma Resusc Emerg Med. 2016;24(1):31.

7. Menon DK, Schwab K, Wright DW, Maas AI; Demographics and Clinical Assessment Working Group of the International and Interagency Initiative toward Common Data Elements for Research on Traumatic Brain Injury and Psychological Health. Position statement: definition of traumatic brain injury. Arch Phys Med Rehabil. 2010;91(11):1637-1640.

8. Narayan RK, Michel ME, Ansell B, et al. Clinical trials in head injury. J Neurotrauma. 2002;19(5):503-557.

9. Mehta RL, Kellum JA, Shah SV, et al; Acute Kidney Injury Network. Acute Kidney Injury Network: report of an initiative to improve outcomes in acute kidney injury. Crit Care. 2007;11(2):R31.

10. Sadaka F, Cytron MA, Fowler K, Javaux VM, O'Brien J. Sepsis in the neurologic intensive care unit: epidemiology and outcome. J Clin Med Res. 2015;7(1):18-20.

11. Vincent JL, de Mendonça A, Cantraine F, et al. Use of the SOFA score to assess the incidence of organ dysfunction/ failure in intensive care units: results of a multicenter, prospective study. Working group on "sepsis-related problems" of the European Society of Intensive Care Medicine.. Crit Care Med. 1998;26(11):1793-1800.

12. Lang RM, Badano LP, Mor-Avi V, et al. Recommendations for cardiac chamber quantification by echocardiography in adults: an update from the American Society of Echocardiography and the European Association of Cardiovascular Imaging. J Am Soc Echocardiogr. 2015;28(1):1-39.e14.

13. Hasanin A, Kamal A, Amin S, et al. Incidence and outcome of cardiac injury in patients with severe head trauma. Scand J Trauma Resusc Emerg Med. 2016;24(1):58.

14. Krishnamoorthy V, Sharma D, Prathep S, Vavilala MS. Myocardial dysfunction in acute traumatic brain injury relieved by surgical decompression. Case Rep Anesthesiol. 2013;2013:1-4.
15. Chengode S. Left ventricular global systolic function assessment by echocardiography. Ann Card Anaesth. 2016;19(5)(Supplement):26.

16. Blanc C, Zeller M, Cottin Y, et al. Takotsubo Cardiomyopathy Following Acute Cerebral Events. Eur Neurol. 2015;74(34):163-168.

17. Cheah CF, Kofler M, Schiefecker AJ, et al. Takotsubo Cardiomyopathy in Traumatic Brain Injury. Neurocrit Care. 2017;26(2):284-291.

18. Levitov A, Frankel HL, Blaivas M, et al. Guidelines for the Appropriate Use of Bedside General and Cardiac Ultrasonography in the Evaluation of Critically Ill PatientsPart II. Crit Care Med. 2016;44(6):1206-1227.

19. Gudmundsson P, Rydberg E, Winter R, Willenheimer R. Visually estimated left ventricular ejection fraction by echocardiography is closely correlated with formal quantitative methods. Int J Cardiol. 2005;101(2):209-212.

20. Lim HB, Smith M. Systemic complications after head injury: a clinical review. Anaesthesia. 2007;62(5):474-482.

21. Grote S, Böcker W, Mutschler W, Bouillon B, Lefering R. Diagnostic value of the Glasgow Coma Scale for traumatic brain injury in 18,002 patients with severe multiple injuries. $\mathbf{J}$ Neurotrauma. 2011;28(4):527-534.

22. Cinotti R, Piriou N, Launey Y, et al. Speckle tracking analysis allows sensitive detection of stress cardiomyopathy in severe aneurysmal subarachnoid hemorrhage patients. Intensive Care Med. 2016;42(2):173-182.

\section{Author Affiliations}

Chakradhar Venkata, MD*and Jan Kasal, MD, FCCM†

*Department of Critical Care Medicine, Mercy Hospital and Division of Pulmonary and Critical Care Medicine, St. Louis University School of Medicine, St. Louis, Missouri USA †Department of Anesthesia and Critical Care Medicine, Washington University, St. Louis, Missouri USA 